\title{
NONLINEAR DEVICES ACTING AS SNR AMPLIFIERS FOR A HARMONIC SIGNAL IN NOISE*
}

\author{
François Chapeau-Blondeau ${ }^{1}$ and David Rousseau ${ }^{1}$
}

\begin{abstract}
A harmonic signal corrupted by an additive white noise is processed by an arbitrary memoryless nonlinear device. The transformation of the signal-to-noise ratio (SNR) by the nonlinearity is explicitly computed and analyzed for Gaussian and non-Gaussian noise. Simple nonlinearities, easily implementable as electronic circuits, are shown capable of producing an amplification of the SNR. Such an amplification is not obtainable with linear filters, whatever their complexity or high order, but becomes easily accessible with simple nonlinear devices.
\end{abstract}

Key words: Nonlinear device, harmonic signal, amplification, signal-to-noise ratio.

\section{Introduction}

Harmonic signals are very often encountered as a basic component in many situations of signal processing, information coding, and measurement. The harmonic component itself can be the (fixed) signal of interest, or it can be a carrier conveying useful information through modulation of some of its parameters. It is therefore a very common and important problem in many areas of experimental sciences and technologies to have to recover a harmonic signal hidden in additive noise.

A harmonic signal of frequency $v_{s}$ added to a noise constitute a signal-noise mixture with a very characteristic signature in the frequency domain: its power spectrum is formed by a sharp spectral line at the harmonic frequency $v_{s}$, emerging out of a broadband background contributed by the noise. To quantify the relative parts of the harmonic signal and of the noise in the mixture, a signalto-noise ratio (SNR) $\mathcal{R}$ is conveniently defined from the power spectrum, as the ratio of the power contained in the spectral line at $v_{s}$ divided by the power

* Received May 28, 2005; revised October 14, 2005.

${ }^{1}$ Laboratoire d'Ingénierie des Systèmes Automatisés (LISA), Université d'Angers, 62 avenue Notre Dame du Lac, 49000 Angers, France. E-mail for Chapeau-Blondeau: chapeau@univangers.fr; E-mail for Rousseau: david.rousseau@univ-angers.fr 
contained in the noise background in a small reference frequency band $\Delta B$ around $v_{s}$. This SNR measures how well the spectral line at $v_{s}$ emerges from the noise background.

A narrowband filter at $v_{s}$ used to extract the harmonic component will have an efficacy directly increasing with this SNR $\mathcal{R}$ [9]. It may therefore be desirable, as a preprocessing step, to be able to increase this SNR $\mathcal{R}$. Yet, it is known that no linear filter is able to improve (increase) such an SNR $\mathcal{R}$. This is because a linear filter multiplies both the spectral line and the noise background at $v_{s}$ by the same factor, the squared modulus of its transfer function at $v_{s}$ [9]. As a consequence, any linear filter, whatever its complexity or its high order, leaves the SNR $\mathcal{R}$ unchanged.

In contrast, here we will consider simple nonlinear devices, especially easily realizable as electronic circuits. We will demonstrate that these nonlinear devices can act as SNR amplifiers capable of producing an enhancement of the SNR $\mathcal{R}$. One such type of nonlinearity has been investigated in [8] for SNR improvement in Gaussian noise. Here, we will extend the investigation to other types of nonlinearities that we will show to be capable of improving the SNR $\mathcal{R}$. We will also study both Gaussian and non-Gaussian noise conditions, and exhibit the influence of the type of the noise on the improvement of the SNR.

Note that some of the nonlinear devices considered here have also been investigated recently for SNR amplification in the context of stochastic resonance [4]. Stochastic resonance [1], [11] is a nonlinear phenomenon where the presence or even the addition of noise can be exploited, in specific conditions, as a possible means for improving some of the signal processing. Stochastic resonance has been shown exploitable for SNR amplification in the type of memoryless nonlinear devices we will be considering here, and the significance of our results in this direction will also be discussed.

\section{Nonlinear transformation of the SNR}

We consider the signal-noise mixture $x(t)=s(t)+\xi(t)$, with the harmonic component $s(t)=A \cos \left(2 \pi v_{s} t+\varphi\right)$, and with $\xi(t)$ a stationary white noise with cumulative distribution function $F_{\xi}(u)$ and probability density function $f_{\xi}(u)=$ $d F_{\xi}(u) / d u$. This signal $x(t)$ is fed into a memoryless (nonlinear) system [2] with input-output characteristic $g($.) producing the output

$$
y(t)=g[s(t)+\xi(t)] .
$$

In this situation, both $x(t)$ and $y(t)$ are cyclostationary random signals [14] with period $T_{s}=1 / v_{s}$, both showing a power spectrum with a sharp spectral line at $v_{s}$ emerging out of a broadband noise background. The SNR, as defined above, for the output signal $y(t)$ of equation (1), can be expressed in the following way. In the power spectrum of $y(t)$, the power contained in the spectral line at the 
frequency $v_{s}=1 / T_{s}$ is given [5] by $\left|\bar{Y}_{1}\right|^{2}$, where $\bar{Y}_{1}$ is the Fourier coefficient at the fundamental of the $T_{s}$-periodic nonstationary output expectation $\mathrm{E}[y(t)]$, i.e.,

$$
\bar{Y}_{1}=\left\langle\mathrm{E}[y(t)] \exp \left(-\imath \frac{2 \pi}{T_{s}} t\right)\right\rangle,
$$

with the time average defined as

$$
\langle\ldots\rangle=\frac{1}{T_{S}} \int_{0}^{T_{S}} \ldots d t .
$$

In the power spectrum of $y(t)$, the magnitude of the continuous noise background is measured [5] by the stationarized output variance $\langle\operatorname{var}[y(t)]\rangle$, with the nonstationary variance given by $\operatorname{var}[y(t)]=\mathrm{E}\left[y^{2}(t)\right]-\mathrm{E}[y(t)]^{2}$ at a fixed time $t$.

The SNR for the output signal $y(t)$ then results as

$$
\mathcal{R}_{\text {out }}=\frac{\left|\bar{Y}_{1}\right|^{2}}{\langle\operatorname{var}[y(t)]\rangle \Delta t \Delta B},
$$

or equivalently,

$$
\mathcal{R}_{\text {out }}=\frac{\left|\left\langle\mathrm{E}[y(t)] \exp \left(-\imath 2 \pi t / T_{s}\right)\right\rangle\right|^{2}}{\langle\operatorname{var}[y(t)]\rangle \Delta t \Delta B} .
$$

In equations (4)-(5), $\Delta t$ represents the time resolution of the measurement (i.e., the signal sampling period in a discrete time implementation). The white noise assumption here models a broadband physical noise with a correlation duration much shorter than the other relevant time scales, i.e., $T_{s}$ and $\Delta t$, and a finite variance $\sigma_{\xi}^{2}[5]$.

Because the noise $\xi(t)$ is distributed according to $f_{\xi}(u)$, the random signal $x(t)=s(t)+\xi(t)$ at time $t$ is distributed according to $f_{\xi}[u-s(t)]$. Therefore, from equation (1), one obtains the expectations

$$
\mathrm{E}[y(t)]=\int_{-\infty}^{+\infty} g(u) f_{\xi}[u-s(t)] d u,
$$

and

$$
\mathrm{E}\left[y^{2}(t)\right]=\int_{-\infty}^{+\infty} g^{2}(u) f_{\xi}[u-s(t)] d u .
$$

In a similar way, the SNR for the input signal $x(t)$ is

$$
\mathcal{R}_{\text {in }}=\frac{A^{2} / 4}{\sigma_{\xi}^{2} \Delta t \Delta B} .
$$

The resulting input-ouput SNR gain follows as

$$
G=\frac{\mathcal{R}_{\text {out }}}{\mathcal{R}_{\text {in }}}=\frac{\left|\left\langle\mathrm{E}[y(t)] \exp \left(-\imath 2 \pi t / T_{s}\right)\right\rangle\right|^{2}}{\langle\operatorname{var}[y(t)]\rangle} \frac{\sigma_{\xi}^{2}}{A^{2} / 4} .
$$



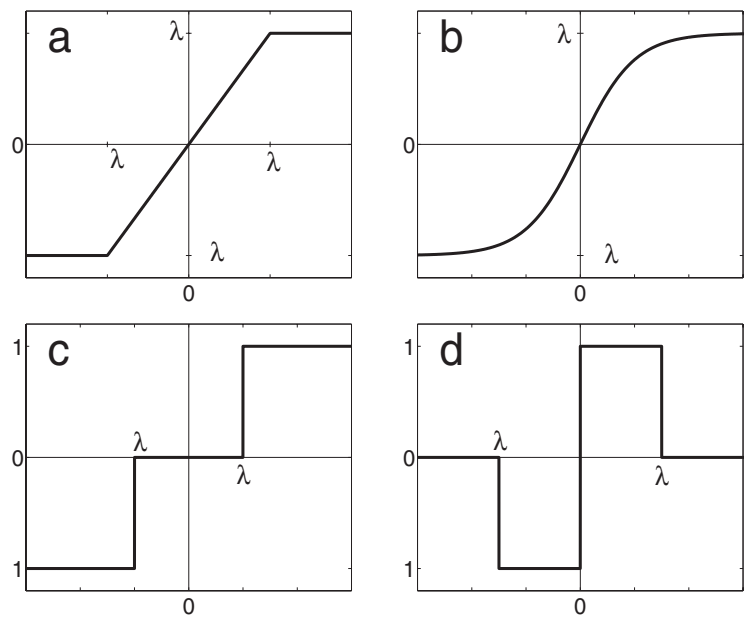

Figure 1. Four simple instances for the nonlinearity $g($.$) in equation (1): (a) hard saturation of$ equation (10), (b) soft saturation of (16), (c) three-level symmetric quantizer of (19), (d) symmetric zeroer of (22).

Equations (3)-(7) provide access to the SNR gain $G$ of equation (9) for an arbitrary nonlinearity $g($.$) and an arbitrary noise density f_{\xi}(u)$. We will now consider four simple nonlinearities $g($.$) , easily implementable with electronic$ circuits, as depicted in Figure 1, and study their impact on the SNR gain $G$ of equation (9). All four nonlinearities in Figure 1 are parameterized by a parameter $\lambda$ that can be optimized to maximize their performing efficacy.

\section{Hard saturation}

We first consider for $g($.$) the hard saturation, depicted in Figure 1a and defined as$

$$
g(u)= \begin{cases}-\lambda & \text { for } \quad u \leq-\lambda \\ u & \text { for }-\lambda<u<\lambda \\ \lambda & \text { for } u \geq \lambda\end{cases}
$$

with the "clipping" parameter $\lambda>0$. 


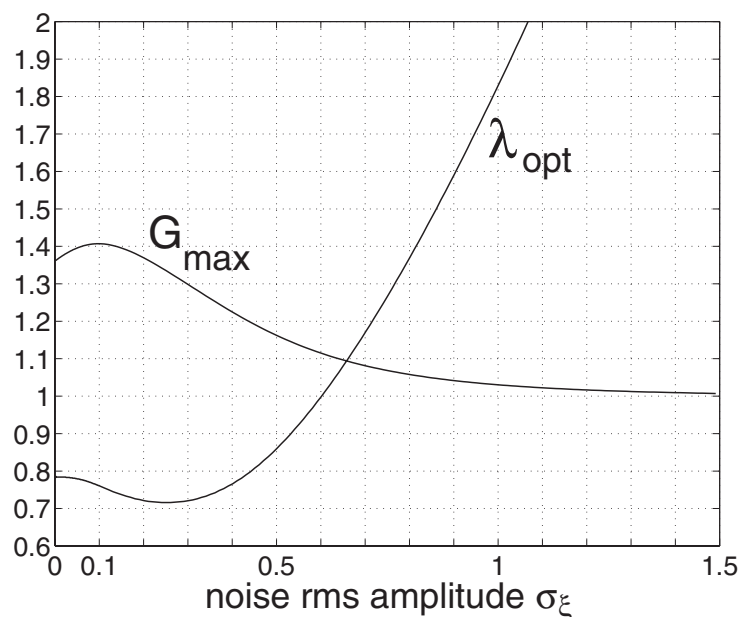

Figure 2. Optimal clipping $\lambda_{\text {opt }}$ in the hard saturation equation (10), and maximum input-ouput SNR gain $G_{\max }$ at $\lambda_{\mathrm{opt}}$, as a function of the rms amplitude $\sigma_{\xi}$ (in units of $A=1$ ) of the zero-mean Gaussian noise $\xi(t)$.

\subsection{Gaussian noise}

When $f_{\xi}(u)$ is a zero-mean Gaussian density associated to the cumulative distribution function $F_{\xi}(u)=\left\{1+\operatorname{erf}\left[u /\left(\sqrt{2} \sigma_{\xi}\right)\right]\right\} / 2$, then equations (6)-(7) give

$$
\begin{aligned}
\mathrm{E}[y(t)]= & \lambda+(-\lambda-s(t)) F_{\xi}(-\lambda-s(t))-(\lambda-s(t)) F_{\xi}(\lambda-s(t)) \\
& +\sigma_{\xi}^{2}\left[f_{\xi}(-\lambda-s(t))-f_{\xi}(\lambda-s(t))\right],
\end{aligned}
$$

and

$$
\begin{aligned}
\mathrm{E}\left[y^{2}(t)\right]= & \lambda^{2}+\left(\lambda^{2}-s^{2}(t)-\sigma_{\xi}^{2}\right)\left[F_{\xi}(-\lambda-s(t))-F_{\xi}(\lambda-s(t))\right] \\
& +\sigma_{\xi}^{2}\left[(-\lambda-s(t)) f_{\xi}(\lambda-s(t))-(\lambda-s(t)) f_{\xi}(-\lambda-s(t))\right] .
\end{aligned}
$$

For the resulting input-ouput SNR gain $G$ of (9), it follows that there is a broad range of values for the clipping parameter $\lambda$, where $G$ is above unity. Furthermore, at each noise level $\sigma_{\xi}$, it is possible to find the optimal clipping $\lambda_{\text {opt }}$ that maximizes the SNR gain $G$. These results are presented in Figure 2.

The results of Figure 2 clearly establish that a simple nonlinear device, like the saturation of equation (10), is capable of yielding an SNR gain larger than unity. This is true for any input noise level $\sigma_{\xi}$, or equivalently for any input SNR $\mathcal{R}_{\text {in }}$, although at very large noise the gain is modest and returns to one from above. The nonlinearity used in Figure 2 thus always delivers an ouput SNR $\mathcal{R}_{\text {out }}$ larger than the input SNR $\mathcal{R}_{\text {in }}$. This is a remarkable outcome, with a simple nonlinear device, 
because a linear device, even very complex (of high order), is incapable of such an SNR amplification.

A qualitative explanation of the operation of the nonlinearity is that the clipping device (10) on the signal-noise mixture $x(t)=s(t)+\xi(t)$ is able to reduce the noise $\xi(t)$ more than it reduces the harmonic signal $s(t)$, this resulting in an inputoutput amplification of the SNR, expressed by $G>1$.

Figure 2 also indicates that the optimal clipping $\lambda_{\text {opt }}$ is not necessarily at the signal amplitude $A$; depending on the noise level, $\lambda_{\text {opt }}$ can be below or above $A$. Also noticeable in Figure 2, is that the optimal clipping $\lambda_{\text {opt }}$ grows to infinity at large noise level $\sigma_{\xi}$, while at the same time the SNR gain returns (from above) to unity. This is in fact the linear behavior in (10) which is recovered as the optimal processor in the large noise limit.

The results of Figure 2 refer to the case of a Gaussian noise $\xi(t)$, which is often met in practice. Yet, in this nonlinear context, it is interesting to examine how the SNR amplification evolves in the case of a non-Gaussian noise $\xi(t)$.

\subsection{Non-Gaussian noise}

When $f_{\xi}(u)$ is an arbitrary density associated to the cumulative distribution function $F_{\xi}(u)$, equations (6)-(7) give

$$
\begin{aligned}
\mathrm{E}[y(t)]= & \lambda+(-\lambda-s(t)) F_{\xi}(-\lambda-s(t))-(\lambda-s(t)) F_{\xi}(\lambda-s(t)) \\
& -G_{\xi}(-\lambda-s(t))+G_{\xi}(\lambda-s(t)),
\end{aligned}
$$

and

$$
\begin{aligned}
\mathrm{E}\left[y^{2}(t)\right]= & \lambda^{2}+\left[\lambda^{2}-s^{2}(t)\right]\left[F_{\xi}(-\lambda-s(t))-F_{\xi}(\lambda-s(t))\right] \\
& -2 s(t)\left[G_{\xi}(-\lambda-s(t))-G_{\xi}(\lambda-s(t))\right] \\
& -H_{\xi}(-\lambda-s(t))+H_{\xi}(\lambda-s(t)),
\end{aligned}
$$

with the functions $G_{\xi}(u)=\int_{-\infty}^{u} v f_{\xi}(v) d v$ and $H_{\xi}(u)=\int_{-\infty}^{u} v^{2} f_{\xi}(v) d v$. Equations (11) and (12), respectively, are a special case of (13)-(14) when the noise $\xi(t)$ is Gaussian.

For illustration, we will consider two different non-Gaussian densities. One has thinner wings compared to the Gaussian; it is for $f_{\xi}(u)$ the uniform density over $\left[-\sqrt{3} \sigma_{\xi}, \sqrt{3} \sigma_{\xi}\right]$. The other one has thicker wings compared to the Gaussian; it is the zero-mean Laplacian density

$$
f_{\xi}(u)=\frac{1}{\sigma_{\xi} \sqrt{2}} \exp \left(-\sqrt{2} \frac{|u|}{\sigma_{\xi}}\right) .
$$

The associated results for the optimal value of $\lambda$ in equation (10) maximizing the SNR gain $G$, along with the corresponding maximum SNR gain, are shown in Figure 3 . 

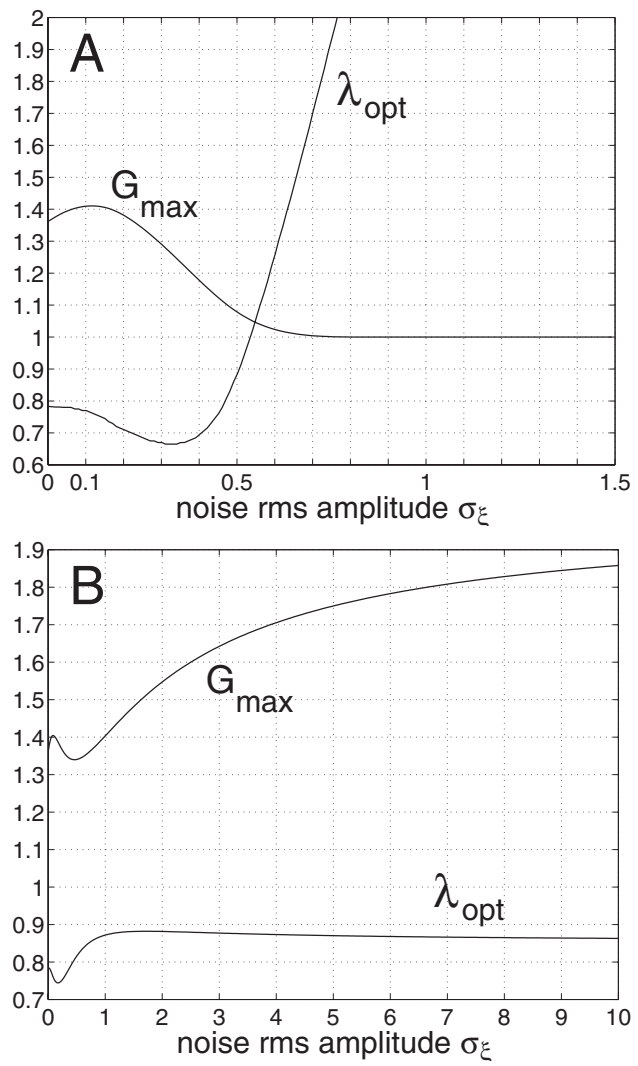

Figure 3. Same as Figure 2, except for the noise density $f_{\xi}(u)$, which is zero-mean uniform (panel A), zero-mean Laplacian (panel B).

The first important observation in Figure 3 is that at the optimal tuning $\lambda_{\text {opt }}$, the SNR gain is always above unity. This means that, for any noise level $\sigma_{\xi}$, the saturating nonlinearity can again act as an SNR amplifier, delivering an ouput SNR $\mathcal{R}_{\text {out }}$ always above the input SNR $\mathcal{R}_{\text {in }}$. This general property of SNR amplification is preserved for the non-Gaussian noises in Figure 3 as well as for the Gaussian noise in Figure 2. It is quite likely, and can be readily verified through evaluation of equation (9), that other choices for the noise density $f_{\xi}(u)$ will generally preserve the possibility of SNR amplification.

Beyond the general preservation of the SNR amplification, it is interesting to note that Figure 3 also reveals some specific influences of the type of the noise $\xi(t)$. With uniform noise, Figure 3(A) shows evolutions much similar to those of Figure 2 with Gaussian noise. At large noise levels $\sigma_{\xi}$, the SNR gain $G_{\max }>1$ returns to one from above; meanwhile $\lambda_{\text {opt }}$ goes to infinity, i.e., the linear behavior in equation (10) is recovered as the optimal processor. With Laplacian noise, 
Figure $3 \mathrm{~B}$ reveals a markedly different picture. At large noise levels $\sigma_{\xi}$, the SNR gain $G_{\max }>1$ grows to saturate around 2, meanwhile $\lambda_{\text {opt }}$ saturates around 0.86. This means that at large Laplacian noise, the linear behavior in (10) is never recovered as the optimal processor; on the contrary, the optimal processor remains strictly nonlinear, and it affords an SNR gain that never returns to one but stabilizes around 2.

A qualitative interpretation of these observations again is based on the nonlinear clipping operation realized by equation (10), which affects the noise more than the signal. The efficient implementation of this clipping, and the effects it yields, strongly depend upon the type of the noise and, especially, the decay of the wings of the noise density $f_{\xi}(u)$ in relation to the Gaussian. Fast or slow decaying wings need be clipped differently for efficient operation. Quantitatively, this is conveyed in the exploitation of equation (9), as shown in Figures 2 and 3.

\section{Soft saturation}

Another common saturating nonlinearity can be tested for SNR amplification and follows the soft saturation model

$$
g(u)=\lambda \tanh (u / \lambda),
$$

depicted in Figure 1(b). Equation (16) is a soft analog of equation (10) and Figure 1a. Both nonlinearities are linear as $g(u) \approx u$ at small $u$, and saturate at $\pm \lambda$ at large $u$.

With $g($.$) of (16) and f_{\xi}(u)$ a Gaussian density (or a Laplacian one), the integrals of equations (6)-(7) have no simple analytical expressions that would parallel (11)-(12); yet numerical integrations can easily be performed, so as to compute the output SNR $\mathcal{R}_{\text {out }}$ of equation (5). This reveals again the feasibility of an SNR gain $G$ above unity, and the possibility of optimizing $\lambda$ in equation (16) so as to maximize the gain $G$. The outcome for the zero-mean Gaussian noise $\xi(t)$ is shown in Figure 4, and for the zero-mean Laplacian noise in Figure 5B.

For $\xi(t)$ a noise uniform over $\left[-\sqrt{3} \sigma_{\xi}, \sqrt{3} \sigma_{\xi}\right]$, analytical expressions are accessible for equations (6)-(7) as

$$
\mathrm{E}[y(t)]=\frac{\lambda^{2}}{2 \sqrt{3} \sigma_{\xi}} \ln \left(\frac{\cosh \left[\left(s(t)+\sqrt{3} \sigma_{\xi}\right) / \lambda\right]}{\cosh \left[\left(s(t)-\sqrt{3} \sigma_{\xi}\right) / \lambda\right]}\right),
$$

and

$$
\mathrm{E}\left[y^{2}(t)\right]=\lambda^{2}+\frac{\lambda^{3}}{2 \sqrt{3} \sigma_{\xi}}\left(\tanh \left[\left(s(t)-\sqrt{3} \sigma_{\xi}\right) / \lambda\right]-\tanh \left[\left(s(t)+\sqrt{3} \sigma_{\xi}\right) / \lambda\right]\right),
$$

yielding the evolutions of Figure 5A.

Figures 4 and 5 reveal that the SNR amplification observed in Figures 2 and 3 


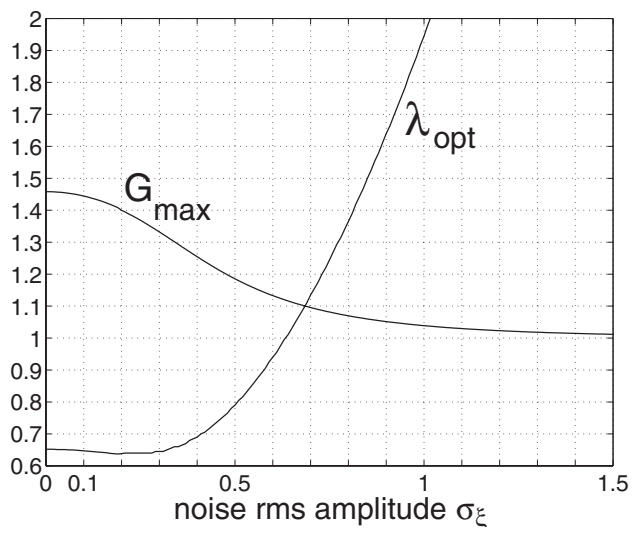

Figure 4. Optimal value $\lambda_{\text {opt }}$ for $\lambda$ in the soft saturation equation (16), and maximum input-ouput SNR gain $G_{\max }$ at $\lambda_{\mathrm{opt}}$, as a function of the rms amplitude $\sigma_{\xi}$ (in units of $A=1$ ) of the zero-mean Gaussian noise $\xi(t)$.

with the hard saturation of equation (10) and Figure 1a, is preserved with the soft saturation of equation (16) and Figure 1b. Moreover, the corresponding evolutions of Figures 4, 5 and of Figures 2, 3 remain quite similar, differing only in minor quantitative details. This similarity of behavior can be attributed to the similarity of the nonlinear devices of Figure 1a and Figure 1b, and expresses some form of robustness in the mechanism of nonlinear amplification of the SNR. In particular, the invertible (Figure 1b) or noninvertible (Figure 1a) character of the nonlinearity $g($.$) is not a critical issue for the SNR amplification.$

\section{Three-level symmetric quantizer}

Another common nonlinearity, easily implementable with electronic devices, is the three-level symmetric quantizer

$$
g(u)= \begin{cases}-1 & \text { for } u \leq-\lambda, \\ 0 & \text { for }-\lambda<u<\lambda, \\ 1 & \text { for } u \geq \lambda,\end{cases}
$$

depicted in Figure 1(c). In this case, equations (6) and (7) yield, respectively,

$$
\mathrm{E}[y(t)]=1-F_{\xi}[\lambda-s(t)]-F_{\xi}[-\lambda-s(t)],
$$

and

$$
\mathrm{E}\left[y^{2}(t)\right]=1-F_{\xi}[\lambda-s(t)]+F_{\xi}[-\lambda-s(t)] .
$$

This leads to the results of Figure 6 when $\xi(t)$ is a zero-mean Gaussian noise. 

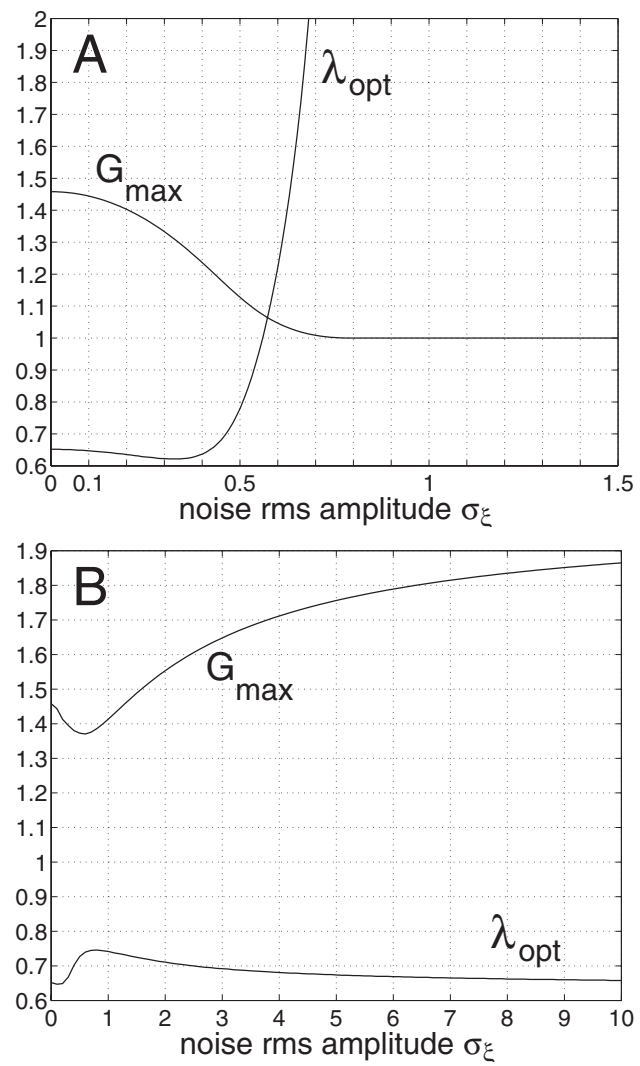

Figure 5. Same as Figure 4, except for the noise density $f_{\xi}(u)$, which is zero-mean uniform (panel A), zero-mean Laplacian (panel B).

An important observation in Figure 6, is that even at the optimal tuning $\lambda_{\text {opt }}$ in (19), the achievable SNR gain always remains below unity. This demonstrates that the property of input-output amplification of the SNR is not realizable with all types of nonlinearities $g($.$) . In this respect, the saturating nonlinearities of$ Figures 1a and $1 \mathrm{~b}$ are capable of an SNR gain $G>1$, whereas the threshold nonlinearity of Figure 1c is not, at least with Gaussian noise.

Figure 7 addresses the situations where $\xi(t)$ is a uniform or Laplacian noise.

The results of Figure 7 indicate that the possibility of an SNR gain above unity is recovered with non-Gaussian noises, at least for sufficiently large noise levels $\sigma_{\xi}$. Also, Figure 7 again illustrates the specific influences that the type of the noise $\xi(t)$ may have on the details of the quantitative evolutions. For instance, with uniform noise, Figure 7A shows an SNR gain $G_{\max }$ growing linearly with the noise level $\sigma_{\xi}$. Because the input SNR $\mathcal{R}_{\text {in }}$ decays as $\sim \sigma_{\xi}^{-2}$, an SNR gain linearly 


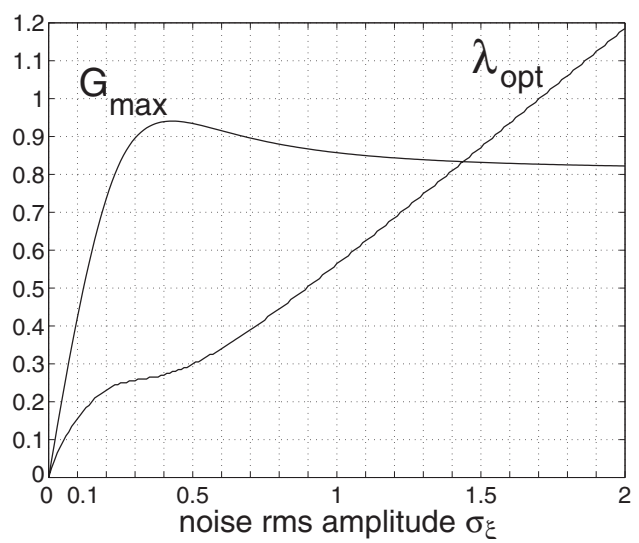

Figure 6. Optimal threshold $\lambda_{\text {opt }}$ for $\lambda$ in the three-level quantizer equation (19), and maximum inputouput SNR gain $G_{\max }$ at $\lambda_{\text {opt }}$, as a function of the rms amplitude $\sigma_{\xi}$ (in units of $A=1$ ) of the zero-mean Gaussian noise $\xi(t)$.

growing as $\sim \sigma_{\xi}$ is equivalent to an output SNR $\mathcal{R}_{\text {out }}$ decaying as $\sim \sigma_{\xi}^{-1}$ when $\sigma_{\xi}$ grows. This indicates that this time at large uniform noise, the threshold nonlinearity of Figure 1c can outperform the saturating nonlinearities of Figures 1a and $1 \mathrm{~b}$ in terms of the SNR $\mathcal{R}_{\text {out }}$ delivered at the output. On a broader level, this also shows the intricate behavior that may result from equation (9) for the SNR gain, depending on the types of the noise $\xi(t)$ and of the nonlinearity $g($.$) ,$ meanwhile ensuring the SNR amplification.

\section{Symmetric zeroer}

To conclude the survey of common nonlinearities with easy practical implementation, we consider the case where the nonlinearity $g($.$) is$

$$
g(u)= \begin{cases}0 & \text { for } \quad u \leq-\lambda, \\ -1 & \text { for } \quad-\lambda<u<0, \\ 1 & \text { for } \quad 0<u<\lambda, \\ 0 & \text { for } \quad u \geq \lambda,\end{cases}
$$

as depicted in Figure 1d. In this case, equations (6) and (7) yield, respectively,

$$
\mathrm{E}[y(t)]=F_{\xi}[\lambda-s(t)]+F_{\xi}[-\lambda-s(t)]-2 F_{\xi}[-s(t)],
$$

and

$$
\mathrm{E}\left[y^{2}(t)\right]=F_{\xi}[\lambda-s(t)]-F_{\xi}[-\lambda-s(t)] .
$$

This leads to the results of Figure 8 for Gaussian noise, and of Figure 9 for uniform or Laplacian noise. 

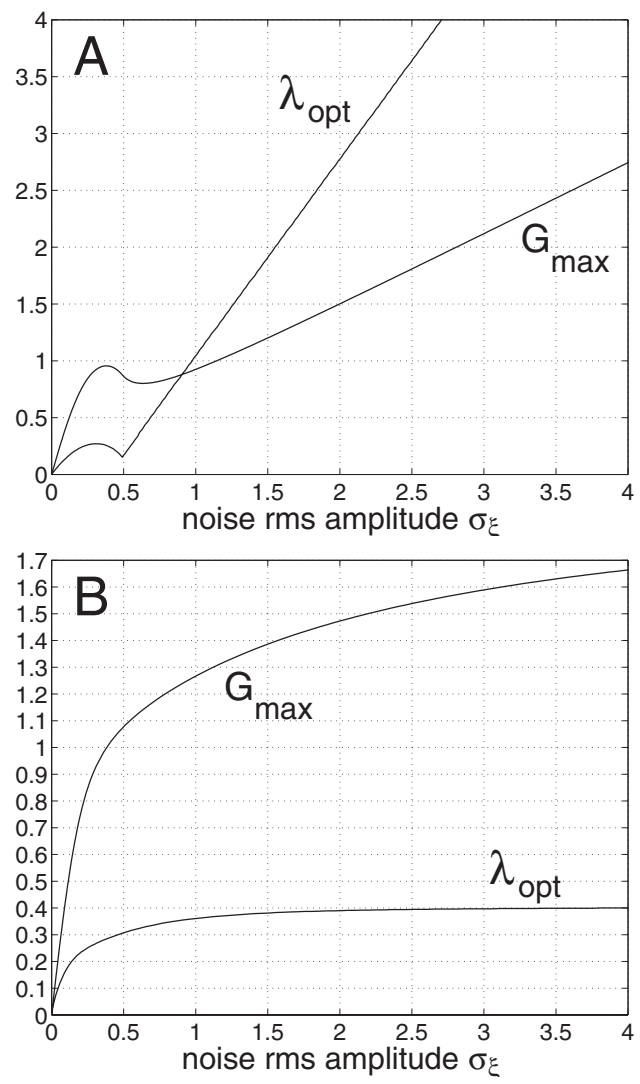

Figure 7. Same as Figure 6, except for the noise density $f_{\xi}(u)$, which is zero-mean uniform (panel A), zero-mean Laplacian (panel B).

The results of Figures 8 and 9 indicate that, in terms of SNR gain, the zeroer nonlinearity of Figure 1d never outperforms the saturating nonlinearities of Figure 1a or 1b. In Figures 8 and 9, in most conditions the SNR gain is below one, except with Laplacian noise at sufficiently large noise levels $\sigma_{\xi}$. Again, the Figures illustrate that not all types of nonlinearities $g($.$) are appropriate to obtain$ the input-output amplification of the SNR, especially with Gaussian noise.

\section{Conclusion}

In this paper we have shown that simple nonlinear devices, easily implementable as electronic circuits, can realize an amplification of the SNR of a harmonic signal in noise. This is a valuable property which is inaccessible to linear devices, what- 


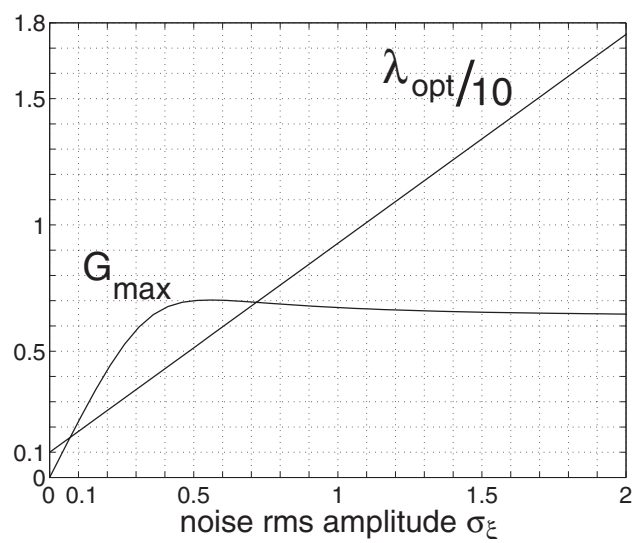

Figure 8. Optimal value $\lambda_{\text {opt }}$ for $\lambda$ in the symmetric zeroer equation (22), and maximum input-ouput SNR gain $G_{\max }$ at $\lambda_{\text {opt }}$, as a function of the rms amplitude $\sigma_{\xi}$ (in units of $A=1$ ) of the zero-mean Gaussian noise $\xi(t)$. It is $\lambda_{\text {opt }} / 10$ that is plotted so as to fit inside the figure.

ever their complexity, and it is remarkable that it becomes feasible with simple nonlinear devices. Such simple nonlinear operators acting as SNR amplifiers offer a useful complement to linear techniques for signal processing and sensors.

Note that we are dealing with static nonlinear devices, which do not impose, by themselves, frequency limitations. As a consequence, SNR amplification is obtained in the same way, in principle, whatever the frequency $v_{s}$ of the harmonic component. In particular, the SNR gain is insensitive to the frequency $v_{s}$ of the harmonic component being amplified. This is a marked difference afforded by static devices, compared to dynamic devices or filters which introduce inherent frequency limitations through their specific time constants. This frequency invariance with static devices is true as long as the white noise assumption remains valid, i.e., as long as the correlation duration of the physical noise remains small in relation to the period $T_{s}=1 / v_{s}$ and other relevant time scales in the process. If the white noise assumption fails, SNR amplification may still be expected, but with a theoretical description that falls outside the scope of equation (9) and that is a priori more complicated to work out. Other natural frequency limitations may arise from the physical realizations, in practice, of the static nonlinearities, for instance, through the use of operational amplifiers with their own cut-off frequencies.

Our study has also shown that the conditions of the SNR amplification are strongly influenced by the type of the noise $\xi(t)$ in addition to the type of the nonlinearity $g($.$) . Not all the conditions of the SNR amplification have been$ worked out exhaustively here; only important illustrative examples have been described. Nevertheless, in principle, all conditions can be explored through the evaluation of equation (9). In this respect, we emphasize that in equation (9) both 

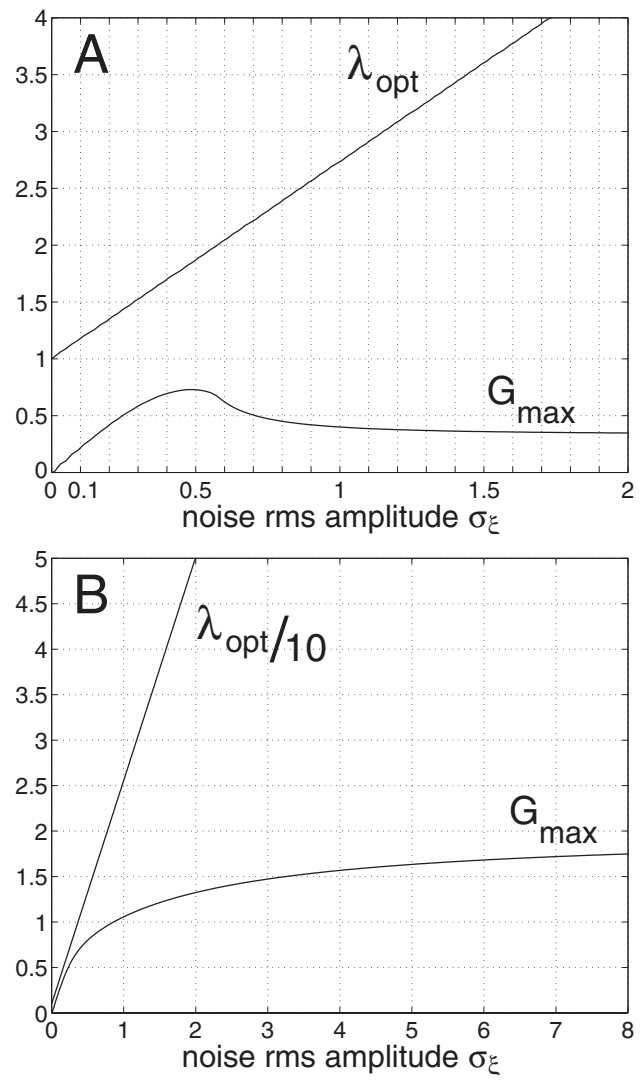

Figure 9. Same as Figure 8, except for the noise density $f_{\xi}(u)$, which is zero-mean uniform (panel A), zero-mean Laplacian (panel B).

the noise probability density $f_{\xi}(u)$ and the nonlinearity $g($.$) are arbitrary, and$ other types of nonlinearity $g($.$) could directly be tested with the present theory$ for SNR amplification.

The SNR amplification of a sinusoid in noise here is based on the selection and optimization of specific static nonlinearities. Another approach has been studied recently for SNR amplification in nonlinear devices, based on the phenomenon of stochastic resonance. So far in this context, it is the static nonlinearities that have been shown to be the most effective (as opposed to dynamic nonlinearities) for SNR amplification of a sinusoid in Gaussian white noise [10], [3], [6]. The two approaches can be contrasted as follows. At a given level of noise $\sigma_{\xi}$, the best thing to do for the SNR, each time it is possible, is to tune the nonlinearity at the optimum $\lambda_{\text {opt }}$. This will ensure the largest input-ouput SNR gain $G$, and consequently the maximally amplified output SNR $\mathcal{R}_{\text {out }}>\mathcal{R}_{\text {in }}$ for a given input 
SNR $\mathcal{R}_{\text {in. }}$. If the nonlinearity cannot be tuned at $\lambda_{\text {opt }}$, then the output SNR $\mathcal{R}_{\text {out }}$ will remain below its maximum. Only in this case of a suboptimal configuration of the nonlinearity can a stochastic resonance effect be envisaged to improve $\mathcal{R}_{\text {out }}$. It will consist of a purposeful addition of noise at the input. Then, in some conditions, especially depending on the type of the noise density $f_{\xi}(u)$ and on the type of the nonlinearity $g($.$) , this may lead to an increase in the output SNR$ $\mathcal{R}_{\text {out }}$. This output SNR could at best reach the maximum SNR authorized by the nonlinearity optimally tuned at the newly increased noise level $\sigma_{\xi}$. But this output SNR would always remain below the SNR that would have been achieved by an optimal tuning of the nonlinearity at the initial noise level $\sigma_{\xi}$, because as can be verified with the present theory, $G_{\max } \mathcal{R}_{\text {in }}$ always decreases when $\sigma_{\xi}$ increases. So each time it is possible, it is the optimal tuning of the nonlinearity, at a fixed given $\sigma_{\xi}$ fixing $\mathcal{R}_{\text {in }}$, which will provide the highest $\mathcal{R}_{\text {out }}>\mathcal{R}_{\text {in }}$. Only when optimal tuning is not feasible, can purposeful addition of noise be tried, for a possible (but not certain, it depends on the conditions) improvement of the output SNR, knowing that the so-produced $\mathcal{R}_{\text {out }}$ is always less than the $\mathcal{R}_{\text {out }}$ that would have resulted from the optimal tuning of the nonlinearity at the initial noise level. This is the picture that emerges based on the current results on stochastic resonance for SNR amplification of a sinusoid in additive Gaussian noise by an isolated static nonlinearity of the type studied here: Addition of noise may possibly lead to an improvement of the output SNR, but only in suboptimal devices, and cannot do better than the optimal device with no added noise. Stochastic resonance by addition of noise can in fact be viewed as a possible (but less efficient) alternative for SNR improvement when the optimization of the nonlinear device is not realizable.

However, the picture concerning the possible benefit of stochastic resonance may evolve, if one considers the possibility of replicating the nonlinear devices $g($.) into parallel arrays, instead of using a single one in isolation. For nonlinearities associated in parallel arrays, an interesting form of stochastic resonance consists in purposely injecting additional noises, on each device of the array, to induce more variability and richness in the responses of the devices [17]. A global response collected over the array of noisy devices can sometimes bring improvement over the performance of a single device with no extra added noise. Various forms of enhanced processing through stochastic resonance in arrays have recently been demonstrated, essentially with two-state quantizers [17], [13], [15] corresponding to the nonlinearity of Figure $1 \mathrm{c}$ tuned at $\lambda=0$. Saturating nonlinearities like those of Figures 1a and $1 \mathrm{~b}$, in terms of SNR gain with Gaussian noise, have been shown here to be superior to threshold nonlinearities like those of Figures 1c and 1d. They are also superior to power-law nonlinearities as tested in [6], and are much more easily implementable with electronic devices. The investigation of such saturating nonlinearities for stochastic resonance in arrays could reveal further useful potentialities for SNR amplification and also for other signal processing tasks [7], [16]. In the same direction, as other interesting nonlinearities to be tested, one could think of basic nonlinearities encountered in semiconductor 
devices or in molecular operators in molecular electronics or nanotechnologies [12]. This together could lead to new generations of smart nonlinear arrays with novel capabilities for information processing.

\section{References}

[1] B. Andò and S. Graziani, Adding noise to improve measurement, IEEE Instrumentation and Measurement Magazine, 4(1):24-30, March 2001.

[2] J. S. Bendat, Nonlinear Systems Techniques and Applications, Wiley, New York, 1998.

[3] J. Casado-Pascual, C. Denk, J. Gómez-Ordóñez, M. Morillo, and P. Hänggi, Gain in stochastic resonance: Precise numerics versus linear response theory beyond the two-mode approximation, Phys. Rev. E, 67:036109, 2003.

[4] F. Chapeau-Blondeau, Input-output gains for signal in noise in stochastic resonance, Phys. Lett. A, 232:41-48, 1997.

[5] F. Chapeau-Blondeau and X. Godivier, Theory of stochastic resonance in signal transmission by static nonlinear systems, Phys. Rev. E, 55:1478-1495, 1997.

[6] F. Chapeau-Blondeau and D. Rousseau, Enhancement by noise in parallel arrays of sensors with power-law characteristics, Phys. Rev. E, 70:060101(R),1-4, 2004.

[7] F. Chapeau-Blondeau and D. Rousseau, Noise-enhanced performance for an optimal Bayesian estimator, IEEE Trans. Signal Process., 52:1327-1334, 2004.

[8] F. Chapeau-Blondeau and D. Rousseau, Nonlinear SNR amplification of a harmonic signal in noise, Electronics Lett., 41:618-619, 2005.

[9] W. B. Davenport and W. L. Root, An Introduction to the Theory of Random Signals and Noise, Wiley, New York, 1987.

[10] P. Hänggi, M. E. Inchiosa, D. Fogliatti, and A. R. Bulsara, Nonlinear stochastic resonance: The saga of anomalous output-input gain, Phys. Rev. E, 62:6155-6163, 2000.

[11] G. P. Harmer, B. R. Davis, and D. Abbott, A review of stochastic resonance: Circuits and measurement, IEEE Trans. Instrumentation Measurement, 51:299-309, 2002.

[12] I. Y. Lee, X. Liu, B. Kosko, and C. Zhou, Nanosignal processing: Stochastic resonance in carbon nanotubes that detect subthreshold signals, Nano Lett., 3:1683-1686, 2003.

[13] M. D. McDonnell, D. Abbott, and C. E. M. Pearce, An analysis of noise enhanced information transmission in an array of comparators, Microelectronics J., 33:1079-1089, 2002.

[14] A. Papoulis, Probability, Random Variables, and Stochastic Processes, McGraw-Hill, New York, 1991.

[15] D. Rousseau and F. Chapeau-Blondeau, Suprathreshold stochastic resonance and signal-tonoise ratio improvement in arrays of comparators, Phys. Lett. A, 321:280-290, 2004.

[16] D. Rousseau and F. Chapeau-Blondeau, Constructive role of noise in signal detection from parallel arrays of quantizers, Signal Process., 85:571-580, 2005.

[17] N. G. Stocks, Information transmission in parallel threshold arrays: Suprathreshold stochastic resonance, Phys. Rev. E, 63:041114,1-9, 2001. 